\title{
HIGH-RESOLUTION ULTRASOUND-AIDED BIOPHOTONIC IMAGING
}

\author{
Lihong V. Wang*, Xueding Wang, Geng Ku, Xueyi Xie, George Stoica \\ Optical Imaging Laboratory, Department of Biomedical Engineering \\ Texas A\&M University, 3120 TAMU, College Station, Texas 77843-3120 \\ URL: http://oilab.tamu.edu; Email:LWang@tamu.edu \\ * Corresponding author.
}

\begin{abstract}
Optical contrast is sensitive to functional parameters, including the oxygen saturation and total concentration of hemoglobin, in biological tissues. However, due to the overwhelming scattering encountered by light in tissues, traditional optical modalities cannot provide satisfactory spatial resolution beyond the ballistic (a few hundred microns) and quasiballistic (1-2 $\mathrm{mm}$ ) regimes. Photoacoustic tomography is based on the high optical contrast yet utilizing the high ultrasonic resolution. Our work in this emerging area of research will be summarized in this invited talk. In this technology, a diffraction-based inverse-source problem is solved in the image reconstruction, for which we developed the rigorous reconstruction theory. We implemented a prototype and accomplished noninvasive transdermal and transcranial functional imaging of small-animal brains in vivo. Change in the cerebral blood oxygenation of a rat, as a result of the alternation from hyperoxia to hypoxia, was imaged successfully.
\end{abstract}

Keywords - Photoacoustic tomography, optical contrast, ultrasonic resolution, inverse-source reconstruction, oxygen saturation of hemoglobin

\section{INTRODUCTION}

Photoacoustic tomography (PAT, also called optoacoustic or thermoacoustic tomography) involves both photons and ultrasound. A short-pulsed laser source is used to irradiate the biological tissue samples under investigation. A temperature rise of the order of $\mathrm{mK}$ will be produced in a short time frame. Consequently, thermoelastic expansion will cause emission of acoustic waves, referred to as photoacoustic waves. The photoacoustic waves are measured by wideband ultrasonic transducers around the sample, and the acquired photoacoustic waves are used to reconstruct the optical absorption distributions. Because the laser pulse is short, proportionately high frequency ultrasonic waves will be produced and used to provide diffraction-limited spatial resolution.

Based on the above principle, the contrast in PAT is determined primarily by the optical properties of the biological tissues, and the spatial resolution in PAT is determined primarily by the photoacoustic waves originating from within the biological tissues. Photoacoustic tomography thereby combines the high optical contrast and the high ultrasonic resolution. This point is summarized in Table 1.

Table 1. Motivation for PAT.

\begin{tabular}{l|lll}
\hline & $\begin{array}{l}\text { All-optical } \\
\text { imaging } \\
\text { (Non- } \\
\text { ballistic) }\end{array}$ & $\begin{array}{l}\text { All-ultrasonic } \\
\text { imaging }\end{array}$ & PAT \\
\hline Contrast & Excellent & $\begin{array}{l}\text { Poor for early } \\
\text { cancers }\end{array}$ & Excellent \\
Resolution & Poor & $\begin{array}{l}\text { Excellent \& } \\
\text { scalable } \\
\text { Good \& } \\
\text { scalable }\end{array}$ & Excellent \\
Depth & Excellent & $\begin{array}{l}\text { Good \& } \\
\text { scalable }\end{array}$ \\
$\begin{array}{l}\text { Speckle } \\
\text { artifacts } \\
\text { Scattering }\end{array}$ & None & Strong & None \\
\hline
\end{tabular}

II. FUNDAMENTALS OF PHOTOACOUSTICS: THE FORWARD PROBLEM

The basic equations in photoacoustic theory will be summarized here. If the electromagnetic pumping pulse duration is much shorter than the thermal diffusion time, thermal diffusion can be neglected; this is known as the assumption of thermal confinement. In this case, the acoustic wave $p(\mathbf{r}, \bar{t})$ is related to electromagnetic absorption, $H(\mathbf{r}, \bar{t})$, by the following wave equation:

$$
\frac{\partial^{2} p(\mathbf{r}, \bar{t})}{\partial \bar{t}^{2}}-\nabla^{2} p(\mathbf{r}, \bar{t})=\frac{\beta v_{s}}{C} \frac{\partial H(\mathbf{r}, \bar{t})}{\partial \bar{t}},
$$

where $\bar{t}=t v_{s} ; v_{s}$ is the acoustic speed, assumed to be constant; $C$ is the specific heat; and $\beta$ is the coefficient of volume thermal expansion. Eq. (1) can be rewritten in 
terms of $H(\mathbf{r}, \bar{t})$ :

$$
p(\mathbf{r}, \bar{t})=\frac{\beta v_{s}}{4 \pi C} \iiint \frac{\partial H\left(\mathbf{r}^{\prime}, t^{\prime}\right)}{\partial t^{\prime}} \frac{d \mathbf{r}^{\prime}}{\left|\mathbf{r}-\mathbf{r}^{\prime}\right|},
$$

where $t^{\prime}=\bar{t}-\left|\mathbf{r}-\mathbf{r}^{\prime}\right|$. The source term $H(\mathbf{r}, \bar{t})$ can further be written as the product of a purely spatial and a purely temporal component, i.e.:

$$
H(\mathbf{r}, \bar{t})=I_{0} \varphi(\mathbf{r}) \eta(\bar{t}),
$$

where $I_{0}$ is a scaling factor proportional to the incident radiation intensity; $\varphi(\mathbf{r})$ describes the electromagnetic absorption properties of the medium at $\mathbf{r}$; and $\eta(\bar{t})$ describes the shape of the irradiating pulse. Substituting Eq. (3) into Eq. (2) results in

$$
p(\mathbf{r}, \bar{t})=\frac{I_{0} \beta v_{s}}{4 \pi C} \iiint \varphi\left(\mathbf{r}^{\prime}\right) \frac{d \eta\left(t^{\prime}\right)}{d t^{\prime}} \frac{d \mathbf{r}^{\prime}}{\left|\mathbf{r}-\mathbf{r}^{\prime}\right|}
$$

This equation provides the solution to the forward problem - prediction of the pressure outside the tissue if the absorption properties of the medium and the profile of the electromagnetic pulse are known. Its inverse problem needs to be solved for imaging.

\section{PHOTOACOUSTIC RECONSTRUCTION: THE INVERSE PROBLEM}

Our group derived the exact inverse solutions in planar, spherical, and cylindrical geometries [1-3]. These exact solutions are computationally intensive and were approximated to more efficient solutions in practical cases [4]. Usually, the distance between the photoacoustic sources and the detector is much longer than the wavelengths of the high-frequency photoacoustic waves that are useful for imaging. Under this far-field condition, the following approximate inverse solution holds:

$$
\varphi(\mathbf{r})=\left.C \iint_{S_{0}} d S_{0} \cos \left(\theta_{d}\right) \frac{1}{t} \frac{\partial p\left(\mathbf{r}_{0}, t\right)}{\partial t}\right|_{t=\left|\mathbf{r}_{0}-\mathbf{r}\right| / v_{s}},
$$

where $C$ is a constant; $S_{0}$ is the surface of detection; and $\theta_{d}$ is the angle between the normal of $d S_{0}$ and $\mathbf{r}-\mathbf{r}_{0}$ (the vector pointing from a point of detection to a point of reconstruction). It should be noted that Eq. (5) is a modified back-projection of quantity $\frac{1}{t} \frac{\partial p\left(\mathbf{r}_{0}, t\right)}{\partial t}$.

This back-projection is analogous to that in $\mathrm{x}$-ray computed tomography $(\mathrm{CT})$. In $\mathrm{x}$-ray $\mathrm{CT}$, the backprojection is along the paths of the x-ray propagation. In PAT, the back-projection however is along the spherical shells that are centered at each of the ultrasonic detectors and that have a radius determined by the acoustic time of flight.

\section{EXPERIMENTS}

The configuration of our PAT system for rat brain imaging is shown in Fig. 1. A dye laser pumped by an Nd:YAG laser provided laser pulses with a tunable wavelength and a 6.5-ns pulse-width. The laser beam was expanded and homogenized before it reached the rat head. The incident energy density of the laser beam was controlled to $<3$ $\mathrm{mJ} / \mathrm{cm}^{2}$ on the surface of the head, which induced a temperature rise in the brain vessels estimated to be $\sim 4 \mathrm{mK}$. The laser-generated photoacoustic waves were coupled into an ultrasonic transducer and were acquired around the head by scanning the ultrasonic transducer around the rat head in the horizontal imaging plane.

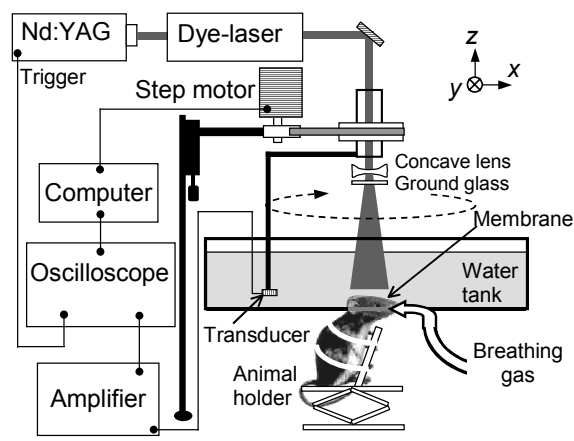

Fig. 1. Prototype PAT for non-invasive transdermal and transcranial imaging of the small-animal brain in vivo with the skin and skull intact.

We used our PAT system to image the cerebral blood oxygenation in a rat brain in vivo. Firstly, pure oxygen $\left(100 \% \mathrm{O}_{2}\right)$ was provided to the rat. In this situation, when the rat was under the hyperoxia status, two photoacoustic images of the optical absorptions in the rat brain cerebral cortex were acquired with the laser light at the 581-nm and the $600-\mathrm{nm}$ wavelengths, respectively [see Figs 2(A) and (B)]. Then the breathing gas was changed slowly from pure oxygen to a mixed gas with a low concentration of oxygen $\left(\sim 8 \% \mathrm{O}_{2}, \sim 5 \% \mathrm{CO}_{2}\right.$ and $\left.\sim 87 \% \mathrm{~N}_{2}\right)$. At this point, when the rat was under the hypoxia status, another two photoacoustic images of the rat brain cerebral cortex, corresponding to the two wavelengths $(581 \mathrm{~nm}$ and $600 \mathrm{~nm}$ ), were acquired [see Figs 2(C) and (D)]. With the high optical contrast between the blood and background brain tissues, each brain image presents the vascular structure in the rat cerebral cortex clearly and matches well with the open-skull anatomical photograph obtained after the imaging experiment [see Fig. 2(H)]. [5].

For each status, the two images of the optical absorptions corresponding to the two wavelengths enable us to simulate the point-to-point hemoglobin oxygen saturation $\left(\mathrm{SO}_{2}\right)$ in the cortical blood vessels through the following equation:

$$
\mathrm{SO}_{2}=\frac{\left[\mathrm{HbO}_{2}\right]}{\left[\mathrm{HbO}_{2}\right]+[\mathrm{Hb}]}=\frac{\mu_{a}^{\lambda_{2}} \varepsilon_{\mathrm{Hb}}^{\lambda_{1}}-\mu_{a}^{\lambda_{1}} \varepsilon_{\mathrm{Hb}}^{\lambda_{2}}}{\mu_{a}^{\lambda_{1}} \varepsilon_{\Delta \mathrm{Hb}^{\lambda_{2}}}-\mu_{a}^{\lambda_{2}} \varepsilon_{\Delta \mathrm{Hb}}^{\lambda_{1}}},
$$


where $\mu_{a}\left(\mathrm{~cm}^{-1}\right)$ is the absorption coefficient; $\lambda_{1}$ and $\lambda_{2}$ are the two wavelengths; $\varepsilon_{\mathrm{Hb}}$ and $\varepsilon_{\mathrm{HbO}_{2}}$ are the known molar extinction coefficients $\left(\mathrm{cm}^{-1} \mathrm{M}^{-1}\right)$ of the oxy- and deoxyhemoglobin, respectively; and $[\mathrm{Hb}]$ and $\left[\mathrm{HbO}_{2}\right]$ are, respectively, the concentrations of the two forms of hemoglobin.

The functional images of the cerebral blood oxygenation corresponding to the hyperoxia and hypoxia statuses are shown in Figs. 2(E) and (F), respectively. These two images use the same gray scale, where darker areas have lower oxygen saturation levels. We can see that when the status of the rat changed from hyperoxia to hypoxia, the blood oxygenation decreased in the rat cerebral cortex. In order to show this change more clearly, we subtracted the image in Fig. 2(E) from the image in Fig. 2(F). The differential image in Fig. 2(G) presents the change of cerebral blood oxygenation when the status of the rat alternated from hyperoxia to hypoxia. The bright areas have negative values, which means that the blood oxygen saturation decreased in the areas of the cortical vessels as a result of this alternation.

\section{SUMMARY}

We developed rigorous reconstruction algorithms, the foundation for PAT. We accomplished non-invasive transdermal and transcranial imaging of small-animal brains in vivo showing both structures and functions. Based on the spectroscopic differences between oxy- and deoxy-hemoglobins, PAT can enable accurate functional imaging of cerebral blood oxygenation in small-animal brains with satisfactory spatial resolution.

\section{ACKNOWLEDGEMENTS}

This project was sponsored in part by the U.S. Army Medical Research and Materiel Command Grant No. DAMD17-00-1-0455, the National Institutes of Health Grants No. R01 EB000712 and No. R01 NS46214, and Texas Higher Education Coordinating Board Grant No. ARP 000512-0063-2001.

\section{REFERENCES}

1. M. Xu and L.-H. Wang, "Time-domain reconstruction for thermoacoustic tomography in a spherical geometry," IEEE Transactions on Medical Imaging 21 (7), 814-822 (2002).

2. Y. Xu, D. Feng, and L.-H. Wang, "Exact frequency-domain reconstruction for thermoacoustic tomography-I: Planar geometry," IEEE Transactions on Medical Imaging 21 (7), $823-828$ (2002).

3. Y. Xu, M. Xu, and L.-H. Wang, "Exact frequency-domain reconstruction for thermoacoustic tomography-II: Cylindrical geometry," IEEE Transactions on Medical Imaging 21 (7), 829-833 (2002).
4. M. Xu, Y. Xu, and L.-H. V. Wang, "Thermo-acoustic imaging in biological tissues: Time-domain reconstruction algorithms", in preparation (2003).

5. X. Wang, Y. Pang, G. Ku, X. Xie, G. Stoica, and L.-H. Wang, "Non-invasive laser-induced photoacoustic tomography for structural and functional imaging of the brain in vivo," Nature Biotechnology 21 (7), 803-806 (2003). 


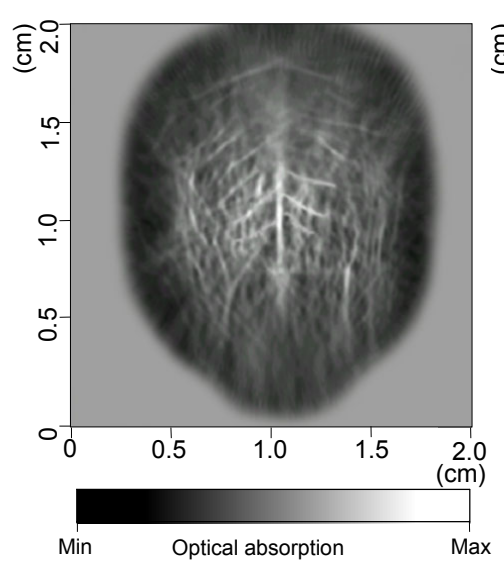

(A)

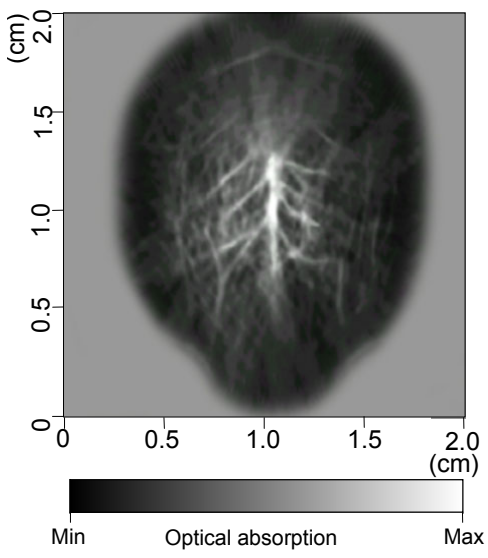

(D)

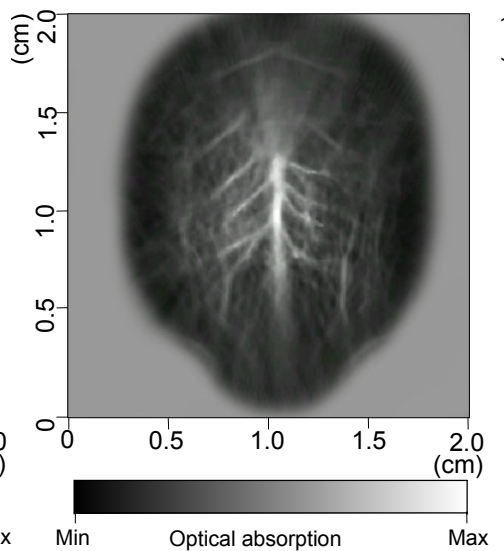

(B)

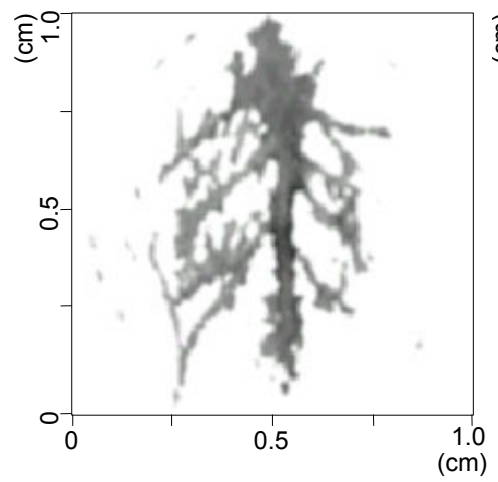

(E)

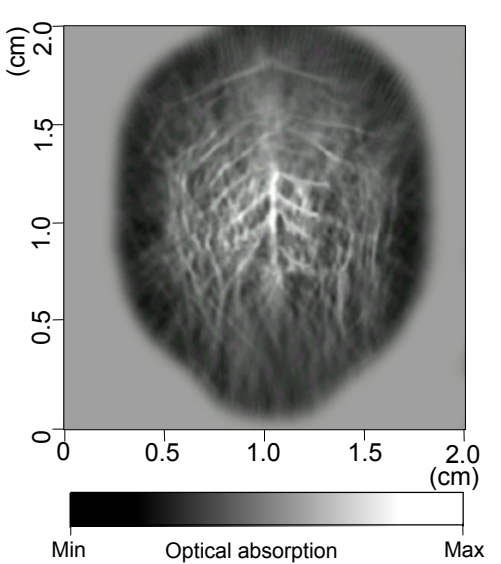

(C)

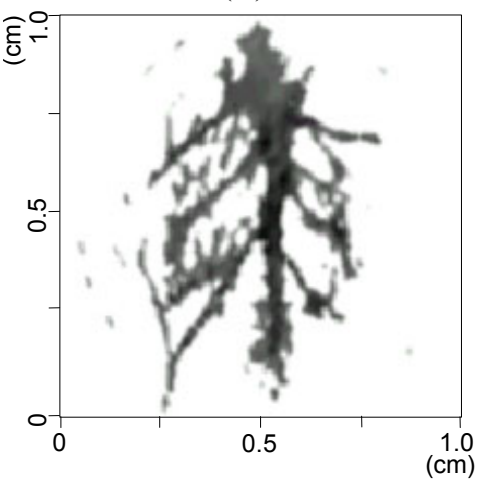

(F)

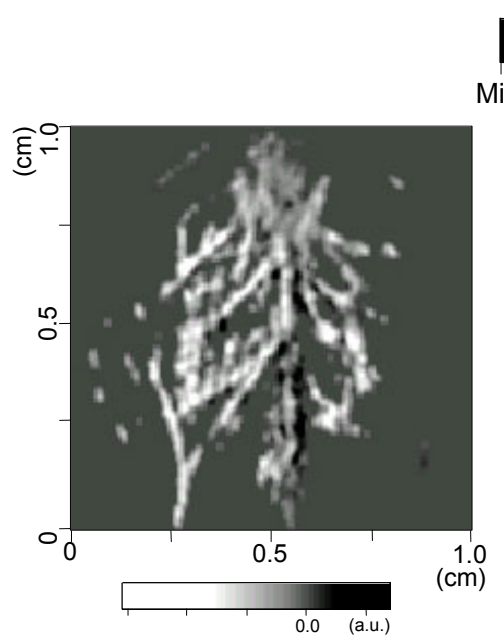

Change of Blood Oxygen Saturation $\left(\Delta \mathrm{SO}_{2}\right)$

(G)
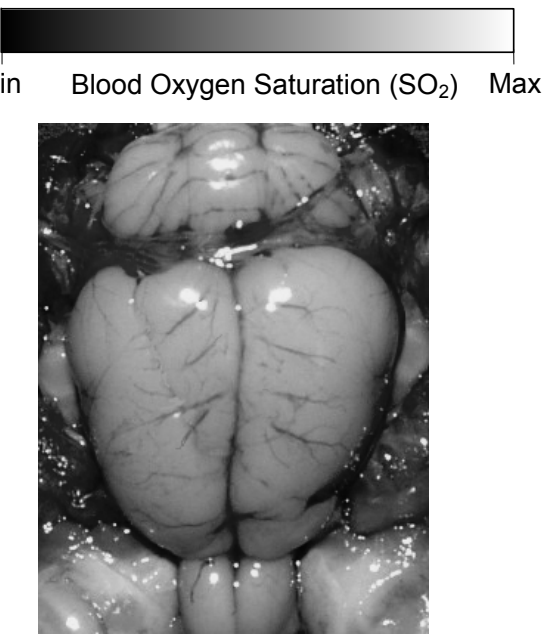

(H)

Fig. 2. Non-invasive functional photoacoustic tomography (PAT) of the cerebral blood oxygenation in a rat brain in vivo. (A) and (B), Brain images under the hyperoxia status corresponding to the $581-\mathrm{nm}$ and the $600-\mathrm{nm}$ wavelengths, respectively. (C) and (D), Brain images under the hypoxia status corresponding to the 581-nm and the 600-nm wavelengths, respectively. (E) and (F), Functional images of the cerebral blood oxygen saturation $\left(\mathrm{SO}_{2}\right)$ in the rat brain under the hyperoxia and hypoxia statuses, respectively. (G) Differential image obtained by F minus E, which presents the change of the blood oxygen saturation in the rat cerebral cortex when the status of the rat alternated from the hyperoxia to the hypoxia. (H) Open-skull anatomical photograph of the rat brain obtained after the imaging experiment. 